\title{
Archival box - a simple tool for the modification of the historic house environments
}

\author{
Morana Novak*a ${ }^{*}$ Josep Grau-Bove ${ }^{\mathrm{a}}$, Caroline De Stefani ${ }^{\mathrm{b}}$, Caroline Checkley-Scott $^{\mathrm{c}}$, Ida Kraševec ${ }^{\mathrm{d}}$, Irena \\ Kralj-Cigićd ${ }^{d}$ Matija Strlič ${ }^{\mathrm{a}}$ \\ a UCL Institute for Sustainable Heritage, London, UK \\ b London Metropolitan Archives, London, UK \\ c Conservation by Design Ltd, Milton Keynes, UK
}

d Faculty of Chemistry and Chemical Technology, Ljubljana, Slovenia

\begin{abstract}
The suitability of archival boxes to mitigate humidity fluctuations in historical houses was investigated. The properties of archival boxes, such as moisture sorption, moisture transmission rates and air exchange rates, were tested. Non-modified boxes made of cardboard respond quickly to humidity changes in storage areas; however, surface modification of cardboards reduces the interaction of a box with the external environment and makes the internal environment more stable. Therefore, archival boxes could present a more sustainable alternative to HVAC systems for management of storage environments in historical houses.
\end{abstract}

Peer-review under the responsibility of the organizing committee of the ICMB21.

Keywords: archival box; microenvironments; historic houses; heritage science

\section{Introduction/Background}

Environmental conditions in historic houses are often unstable with significant fluctuations of both temperature and relative humidity. These fluctuations often exceed the recommended thresholds, as prescribed by environmental standards for heritage collections [1]. As the consequence, sensitive heritage materials may show more pronounced degradation, especially composite objects. Since building conservation concerns make installation of HVAC systems into historical structures difficult, we explore the use of archival boxes as a potentially more sustainable microenvironmental management solution for collections in historical buildings. The efficiency of various box designs and modifications was explored in relation to the potential of mitigating humidity fluctuations in storage spaces.

\section{Objectives}

The aim of our study was to investigate the environmental performance of 35 types of archival boxes. This was done in order to assess how the construction of a box (size, design, cardboard type, content, surface modification) influences its properties, and how these potentially affect the microenvironments within the boxes.

\section{Methodology}

The methodology was based on the investigation of the environmental performance of archival boxes. We measured several properties such as air exchange rate, moisture sorption properties and moisture transmission rates (Figure 1a) and analyzed the performance of a box taking all three measured properties into account.

Air exchange rate (AER) was measured using the gas decay method. A small amount of $\mathrm{CO}_{2}$ was introduced into a box and exponential decay of $\mathrm{CO}_{2}$ in time was measured [2].

Moisture sorption properties, i.e. moisture diffusion and moisture isotherms, were investigated using dynamic vapor sorption for each archival board type.

Moisture transmission rates (MTR) were measured using standardized wet cup testing [3].

${ }^{*}$ Corresponding author. Mobile: 0044 (0)7746011217. E-mail: morana.novak.17@ucl.ac.uk

(C) The Author(s). This is an open access article distributed under the terms of the Creative Commons Attribution License (CC BY) 4.0 https:// creativecommons.org/licenses/by/4.0/, which permits unrestricted use, distribution and reproduction in any medium, provided the original author and source are credited. DOI: 10.14293/ICMB210043 
ICMB21

Microenvironmental monitoring was done in fluctuating RH and temperature conditions in order to explore how a box microenvironment adjusts to changes in the external environment.

In addition, a simple model, based on the mass transfer phenomena, was developed. Model parameter $s$ describes several processes which happen inside a paperboard, as it interacts with moisture.

\section{Results and discussion}

\subsection{Air exchange rates}

On the basis of the AER experiments, it was concluded that some archival boxes have high AERs, especially those of unmodified cardboard. This can cause rapid moisture ingress, which may render such a box unsuitable for environments with pronounced fluctuations of RH. Modification of the box surface using impermeable coatings lowers the AER, while closing of box holes/openings does not have a significant influence.

\subsection{Moisture sorption properties}

A comparison of sorption isotherms shows slight variations in the behavior of different cardboards, meaning that different boxes will absorb different amounts of moisture in the same environment. Moisture diffusion coefficients also depend on the individual cardboard.

\subsection{Moisture transmission rates}

Measurements of moisture transmission rates (MTR) showed different behaviors related to the rate of moisture transfer, with thinner and non-modified materials having the highest values. Surface modification successfully reduced moisture transmission, leading to better protection against humidity ingress.

\subsection{Microenvironmental monitoring}

The comparison between external environments and microenvironments within boxes showed that a typical archival box rapidly responds to changes in environmental humidity. This is in the agreement with AER and MTR measurements, which show that cardboard rapidly interacts with environmental humidity, which is not surprising, giving that papers is a hydrophilic porous material. Nevertheless, it was shown that surface modification, or stacking of boxes during storage, can efficiently reduce the rates of water exchange with external environments (Figure 1b).
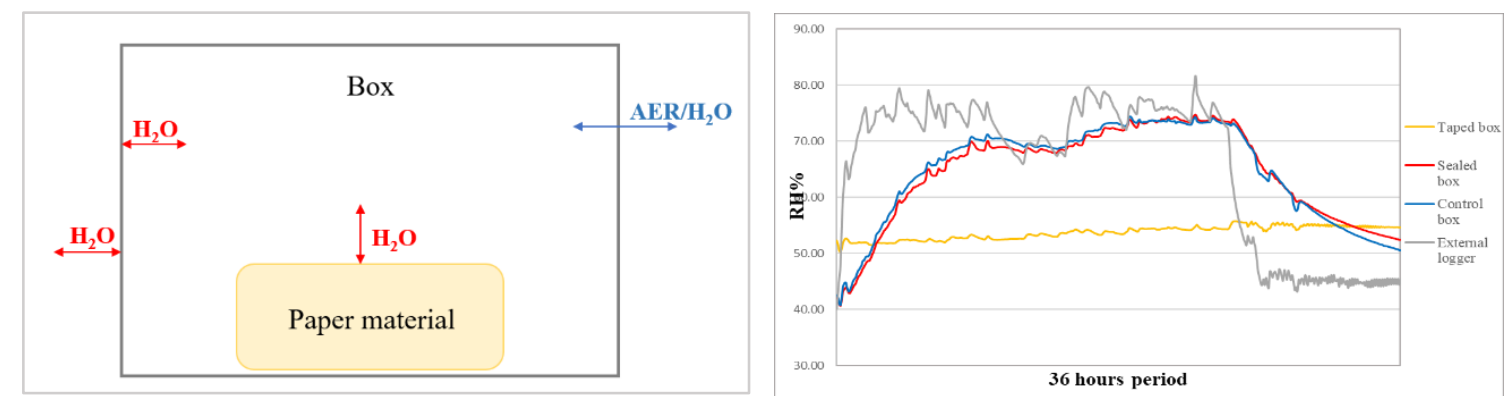

Figure 1: (a) Conceptual model of a box microenvironment; (b) Changes in the microenvironments within selected boxes as a consequence of the changes in the external environment.

\section{Conclusions}

Typical archival boxes interact with external environments rapidly, as evidenced by high air exchange rates, moisture sorption properties and high moisture transmission rates. However, modification of box properties, such as surface coatings can efficiently stabilize microenvironments within boxes. Optimized boxes may be a sustainable storage solution for collections in fluctuating environments. 
1st International Conference on Moisture in Buildings (ICMB21), UCL London 28-29 June 2021

ICMB21

References

[1] Thomson G. The Museum Environment. 2nd Edition. Routledge, New York, USA; 2011

[2] Calver A, Holbrook A, Thickett D, Weintraub S. Simple methods to measure air exchange rates and detect leaks in display and storage enclosures. In: 14th Triennial Meeting, The Hague Preprints; 2005:597-609.

[3] ASTM E96 standard: Cup Method Water Vapor Permeability Testing, 2016. 